\title{
Electromagnetic Simulation of Signal Distribution of Various RF Endoluminal Loop Geometries with Coil Orientation: Towards a Reconfigurable Design
}

\author{
Hamza Raki $\mathbb{D}^{1,2,3}$ Kevin Tse Ve Koon ${ }^{1}{ }^{1},{ }^{1}$ Henri Souchay, ${ }^{3}$ Fraser Robb ${ }^{1 D},{ }^{4}$ \\ and Olivier Beuf ${ }^{1}{ }^{1}$ \\ ${ }^{1}$ Univ Lyon, INSA-Lyon, Université Claude Bernard Lyon 1, UJM-Saint Etienne, CNRS, Inserm, CREATIS UMR 5220, \\ U1294, F-69621, Villeurbanne, France \\ ${ }^{2}$ CRESITT Industrie, Orleans, France \\ ${ }^{3}$ GE Healthcare, Buc, France \\ ${ }^{4}$ GE Healthcare, Cleveland, Ohio, USA \\ Correspondence should be addressed to Olivier Beuf; olivier.beuf@creatis.insa-lyon.fr
}

Received 12 October 2020; Revised 30 June 2021; Accepted 13 July 2021; Published 28 July 2021

Academic Editor: Mazin Jouda

Copyright $\odot 2021$ Hamza Raki et al. This is an open access article distributed under the Creative Commons Attribution License, which permits unrestricted use, distribution, and reproduction in any medium, provided the original work is properly cited.

With the objective of improving MR endoluminal imaging of the colonic wall, electromagnetic simulations of different configurations of single-layer and double-layer, and double-turn endoluminal coil geometries were run. Indeed, during colon navigation, variations in coil orientation with respect to $\mathrm{B}_{0}$ are bound to occur, leading to impaired image acquisition due to a loss of signal uniformity. In this work, three typical coil orientations encountered during navigation were chosen and the resulting signal uniformity of the different geometries was investigated through the simulated $\left(B_{1 x, y} / I \sqrt{R_{t}}\right)$ values. Sampling this quantity over a circle of radius $r$ enabled us to calculate the coefficient of variation ( $=$ standard deviation/mean) for this given distance. This procedure was repeated for $r \in[5 ; 15] \mathrm{mm}$, which represents the region of interest in the colon. Our results show that single-loop and double-layer geometries could provide complementary solutions for improved signal uniformity. Finally, using four microelectromechanical system switches, we proposed the design of a reconfigurable endoluminal coil able to switch between those two geometries while also ensuring the active decoupling of the endoluminal coil during the RF transmission of an MR experiment.

\section{Introduction}

The main inflammatory bowel diseases are ulcerative colitis and Crohn's diseases [1]. They affect the colon and/or rectum with an increased risk to evolve into colorectal cancer (CRC) [2], which is one of the most common cancers $[3,4]$. Worldwide, it is the second and third most frequent cancer for women and men, respectively [5], and accounts for $8.5 \%$ of all cancer deaths [6]. The treatment of CRC at early stages offers a 5 -year survival rate greater than $90 \%$, in contrast to late stages where this rate is less than $10 \%$ [7]. Therefore, it is important to develop new imaging devices or techniques able to provide an accurate diagnosis for each stage, particularly the early stage.
To this end, magnetic resonance imaging (MRI) is one of the major techniques used for medical diagnosis thanks to several developments to improve significantly the signal-tonoise ratio (SNR) by increasing the static magnetic field strength $[8,9]$, or increasing the number of receiver channels and using surface arrays coils [10]. Unfortunately, all these developments are still insufficient for bowel and colon wall imaging. Analysis of the deep and thin colon wall layers as well as assessment and staging of colorectal cancer remain very challenging [11].

Previous works have demonstrated the value of endoluminal imaging based on miniature internal receiver surface coils (endoluminal coils). In the context of colon imaging, endoluminal coils provide a high local SNR very 
close to the region of interest compared with the external surface coils [12]. Preclinical endoluminal coil designs, usually based on single-channel and single-loop geometry, were assessed for imaging of the arterial wall of large vessels [13], the pelvis and gastrointestinal tract [14], rabbit colon wall [15], and mouse colon wall [16]. In clinical routine, endorectal single MR loop coils were used to visualize only the prostate and rectal area (Medrad Prostate eCoil, Bayer) or the cervix (Medrad Cervix eCoil, Bayer). In the context of designing optimal and dedicated coil geometries to improve the radial signal uniformity, an anal-sphincter endoluminal coil based on two turn loops with an angle of $50^{\circ}$ or $70^{\circ}$ between them was assessed. Compared with a single loop, this design made it possible to improve the radial distribution uniformity [17].

An important issue with the use of endoluminal coils and for which no compromise can be made concerns the possible local heating and thus a risk of burn for the patient undergoing such an examination. The use of conventional coaxial cables in the MRI environment induced currents flowing in the external ground of cables leading to an increased local SAR (specific absorption rate) [18]. To circumvent this effect, traps are placed in coaxial cables to reduce the common-mode radiofrequency (RF) current of the cable shield. In most cases in MRI, multiple traps are placed at least at each quarter of wavelength to reduce the coupling. Traps have to handle heating due to a possibly strong $E$ field, and miniaturized devices are not recommended. Besides, they have to be isolated from human tissue by at least 1 or $2 \mathrm{~mm}$ of plastic material. Usually, traps used in the clinic are not very flexible and have a diameter of at least $2 \mathrm{~cm}$, making them unsuitable for endoluminal applications. Thus, a full optical-based signal transmission is a potential alternative to solve this problem [19].

A second limitation is the acceptance and comfort of the patient. In our case, the reduction of the coil diameter $(<10 \mathrm{~mm})$ strongly contributes to advances in this domain by increasing patient comfort. In addition, it also helps obtain a higher local SNR. However, the gain in sensitivity is only local and is accompanied by a rapid reduction with increasing distance from the coil [20]; this is a characteristic of the surface coil family. Fortunately, the targeted colon wall area is approximately $1 \mathrm{~cm}$ thick; rendering endoluminal coil designs a feasible alternative for colon wall imaging.

Several studies were dedicated to endoluminal coils but, to the best of our knowledge, none of the published works addressed sensitivity variations that can be met during navigation within the colon due to coil orientation. This article deals with the problematic related to the required coil navigation within the colon. A loop coil suffers from sensitivity map variations as a function of coil orientation with respect to the main magnetic field $\left(B_{0}\right)$. A maximum of coil sensitivity and uniformity is ensured when the long axis is aligned with $B_{0}$ yielding a normal to the loop coil surface (main $B_{1}$ direction) orthogonal to $B_{0}$ [21]. This orientation is considered as the reference orientation. Departure from this optimal condition induces changes in the coil sensitivity map and thus both intensity values and radial distribution uniformity (shape) are altered by this coil orientation effect, leading to degraded coil sensitivity and consequently impaired image quality. This question of variations in sensitivity associated with orientation is a major challenge for colon wall imaging. As can be seen, the sensitivity variation depends on the colon region and multiple different coil orientations can occur. To simplify this complex problem, as a first step, in this work, we defined three main groups of regions according to the three main coil orientations (see Figure 1):

(i) Vertical regions: rectum (1), descending colon (3), and ascending colon (7)

(ii) Horizontal region: middle part of transverse colon (5)

(iii) Tilted regions: sigmoid (2) and the two extremities of the transverse colon $(4,6)$

Thus, it is necessary to modify the loop geometry according to its orientation. To accomplish this, MR switches can be used. A few years ago, microelectromechanical systems (MEMS) were introduced by GE Healthcare Inc. [22] to act as MR switches. MEMS switches were successfully used (i) to open or close portions of metallic conductors and thus to reconfigure loop coil geometry [23], (ii) to shift the resonance frequency in the case of dual-tuned RF coils $[24,25]$, (iii) to get high RF shimming performances (high localized $B_{0}$ homogeneity) [26], (iv) to control and automatize a wireless power transfer system [27], and finally (v) to control multiple receiver coil arrays with reduced power consumption and cabling system improving the SNR [28]. A recent work [29] demonstrated the feasibility and the impact of using MEMS switches on endoluminal coils for fulfilling the task of serial and parallel active decoupling and studied their impact on the image quality.

In this context, the main goal of the current work was to investigate different loop coil geometries within a small width $(5 \mathrm{~mm})$ and to assess the radial sensitivity pattern as a function of the coil orientation with respect to $B_{0}$ (signal intensity and distribution). This was carried out through electromagnetic (EM) numerical simulations. Then, complementary geometries were chosen to design a theoretically reconfigurable RF coil using switches such as MEMS placed in parallel or in series within the loop path. The latter make it possible to change the coil geometry in view of having an optimal coil sensitivity pattern for each coil orientation selected.

\section{Materials and Methods}

As seen in Figure 2, different miniature rectangular singleand double-loop geometries were defined and evaluated. The dimensions were chosen taking into consideration the eventual insertion into the colon (less than $10 \mathrm{~mm}$ in outer diameter) and defined within a cuboid volume with dimensions of $5 \mathrm{~mm} \times 5 \mathrm{~mm} \times 47 \mathrm{~mm}$ (Figure 2(a)). All loop geometries were designed with $47 \mathrm{~mm}$ conductor strip lengths in $\mathrm{yOz}$ plane.

A diagonal single loop (DSL) was defined in the diagonal (with two opposite parallelepiped faces); thus, the loop width was approximately $7.1 \mathrm{~mm}$ (see Figure 2(b)), and the current 


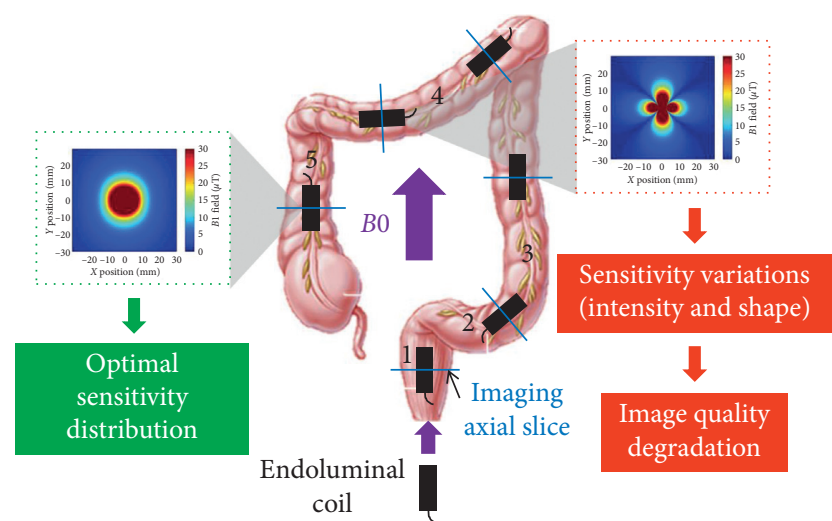

FIgURE 1: The coil path inside the colon: the effect of different coil orientations within $\mathrm{B}_{0}$ on the sensitivity map of the coil. Parts 1, 2, 3 , 4 , and 5 are the rectum, sigmoid colon, descending colon, transverse colon, and ascending colon, respectively.

path is illustrated in Figure 3. Four rectangular double-loop (RDL) geometries were defined with a strip conductor in each parallelepiped face (see Figure 2(c)). Loops were defined on two opposite faces with $5 \mathrm{~mm}$ loop spacing. Then, the current direction was modified to get four possible designs: two doubleturn loops and two double-layer loops. In the double-turn design, the two loops are in series: rectangular double turn with the same (RDT-SC, Figure 4(a)) or opposite (RDT-OC, Figure 4(b)) current direction, whereas for the double-layer design, the loops are connected in parallel: rectangular double layer with the same (RDL-SC, Figure 4(c)) or opposite (RDLOC, Figure 4(d)) current direction. For the two geometry designs, the current can flow in the same direction or in the opposite direction, giving the four geometries studied here. Finally, similarly to the RDL design, a diagonal double loop (DDL) was defined with a strip conductor in each parallelepiped face but linked in diagonals (see Figure 2(d)) to form doubleturn or double-layer loops. The two defined loops are then perpendicular to each other, and the current can flow in two opposite directions: D1 and D2, as shown in Figure 5. Hence, the number of loops, the serial or parallel type, and the current flow direction define each loop coil configuration: diagonal double turns with a first (DDT-D1, Figure 5(a)) or a second (DDT-D2, Figure 5(b)) current direction and diagonal double layers with a first (DDL-D1, Figure 5(c)) or a second (DDL-D2, Figure 5(d)) current direction.

All geometries were first defined using the commercial electromagnetic (EM) software FEKO (EM Software and Systems, South Africa) [30]. Without any coil rotation, we assume that the coil coordinates system $(u, v, n)$ and the MR scanner coordinates system $(x, y, z)$ are superimposed ( $z$ is the $B_{0}$ axis and $x$ is the vertical axis). As shown in Figure 2, all copper strips were drawn with their long axis aligned along $n_{\text {axis }}$. The $u O v$ plane corresponds to the transverse plane of the MR scanner when the coil is in the reference orientation (without any coil rotation). Simulations were carried out based on the full-wave numerical method of moment (MoM) with an arbitrary excitation source of $1 \mathrm{~W}$ power, $1 \mathrm{~V}$ voltage $[31,32]$. Therefore, after defining such loop geometry, a meshing of the surfaces was carried out without the need for boundary conditions. Thus, $\mathbf{H}_{1}(u, v, n)$ magnetic field components were calculated for each loop geometry in a plane perpendicular to the loop's main axis. In the context of colon MRI, this corresponds to acquiring images of the colon with an imaging plane perpendicular to the local colon axis [33], which is the most relevant imaging plane for analysis of the colon wall.

Using Matlab software (Mathworks, Massachusetts, USA), the extracted $\mathbf{H}_{1}$ components were used to deduce the magnetic induction $\mathbf{B}_{1}=\mu \mathbf{H}_{1}$ with the permeability $\mu$ chosen as in the air. The effect of coil orientation with respect to the magnetic field $\mathbf{B}_{0}$ ( $z$-axis) on $\mathbf{B}_{1}(u, v, n)$ components is given by the following $3 \mathrm{D}$ rotation matrices using the following relationship:

$$
B_{1}(x, y, z)=\left[\begin{array}{ccc}
1 & 0 & 0 \\
0 & \cos \alpha & -\sin \alpha \\
0 & \sin \alpha & \cos \alpha
\end{array}\right] \times B_{1}(u, v, n),
$$

where $\alpha$ is the rotation angle about the $x$-axis. To simplify the discussion while being realistic, three coil orientations were studied related to the three groups of regions defined in Figure 1 corresponding to the coil navigation in the colon during an MR examination.

(1) In the vertical regions of the colon, the orientation will be assumed to be $\alpha=0^{\circ}$. Here, we suppose that the coil design including the outer packaging will represent a cylinder with a diameter of $10 \mathrm{~mm}$, which therefore fits tightly within the colon, leaving little possibility for the coils to adopt a different orientation to that of the colon.

(2) The horizontal region of the colon corresponds to an orientation of $\alpha=90^{\circ}$. It should be noted that this is the region where we expect the worst $B_{1}$ uniformity since in this orientation, we anticipate signal cancellation. Again, in this region as in the vertical region, we will suppose that the coils will not be able to adopt a different orientation from that of the colon.

(3) In the tilted regions of the colon, we have assumed that the coil orientation will be $\alpha=45^{\circ}$, which we take to be the highest rotation in these regions and therefore the worst possible case. This is of course a very simplified picture of the different orientations that may occur in the sigmoid or extremities of the transverse colon.

In the imaging procedure, it is assumed that the slice selection orientation will always be orthogonal to the main axis of the coil [33]. Thus, the resulting $B_{1 x, y}$ in the transverse plane $(x O y)$ is given by

$$
B_{1 x, y}=\sqrt{B_{1 x}^{2}+B_{1 y}^{2}} .
$$

Then, SNR distribution can be assessed using the following relationship [34-36]: 


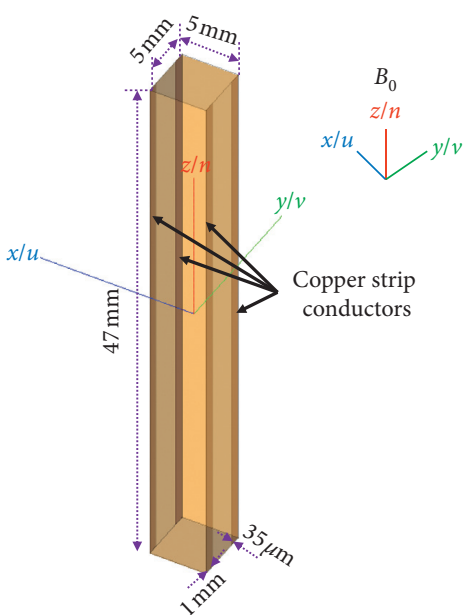

(a)

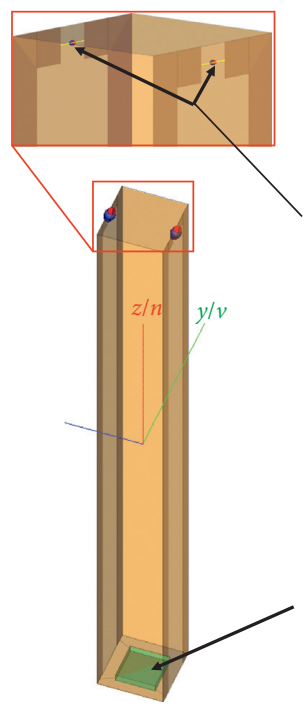

(c)

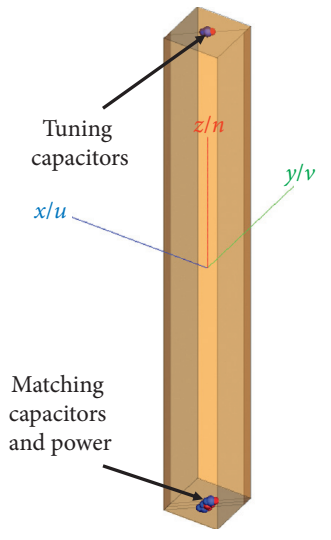

(b)

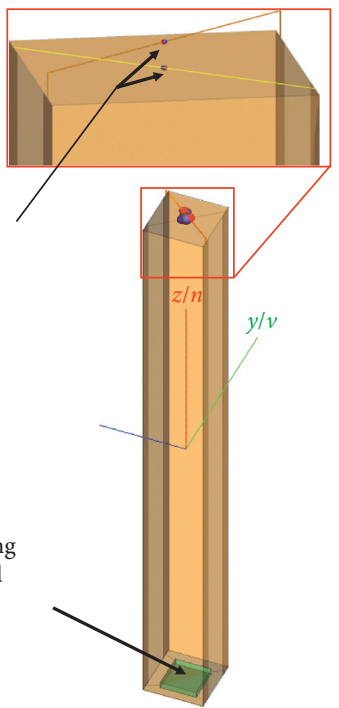

(d)

Figure 2: Different simulated endoluminal loop geometries, defined within (a) a cuboid volume with dimensions of $5 \mathrm{~mm} \times 5 \mathrm{~mm} \times 47 \mathrm{~mm}$, with four copper strip conductors ( $1 \mathrm{~mm}$ width, $47 \mathrm{~mm}$ length, and $35 \mu \mathrm{m}$ thickness). (b) Diagonal single loop (DSL) based on two strips. (c) Rectangular double loop (RDL) based on four strips and thus four current path possibilities. (d) Diagonal double loop (DDL) based on four strips and thus four current path possibilities. It should be noted that the red and blue spheres represent positive and negative poles, respectively, of the capacitors and the excitation port.

$$
\mathrm{SNR}=\frac{\text { peak signal }}{\text { RMS noise }} \propto \frac{B_{1 x y}}{I \sqrt{R_{t}}} .
$$

Here, $I$ is the current flowing in the loop coil and $R_{t}$ is the total resistive loss. Thus, in this work, we will only consider the term $\left(B_{1 x, y} / I \sqrt{R_{t}}\right)$ as a measure of the effect of the reception coil on the SNR.

Both current $I$ and loss $R_{t}$ are estimated by electromagnetic simulations using FEKO software. Adding a very small load $(1 \mathrm{~m} \Omega)$ in series with each loop enables the estimation of the exact current flowing inside each portion of the loop. Loss calculations were derived from the simulated reflection coefficient $\left(S_{11}\right)$ responses in both unloaded and loaded coil states.
To avoid a long simulation time, $S_{11}$ responses were first numerically simulated only at the resonance frequency (63.87 MHz corresponding to a $1.5 \mathrm{~T}$ magnet), and optimal tuning and matching capacitors were found using an optimization method. When getting the best possible matching at this specific frequency, the loop coil was simulated again on a span of $10 \mathrm{MHz}$ frequency. These steps were followed for both unloaded and loaded coils and yielded unloaded and loaded $S_{11}$ responses. The quality factor $Q$ (which is one of the most important quantities used to test the coil performance) in loaded and unloaded conditions was derived from each $S_{11}$ response at a bandwidth of $-3 \mathrm{~dB}[37,38]$. Loss effects $\left(R_{t}\right)$ were then estimated from the simulated unloaded and 


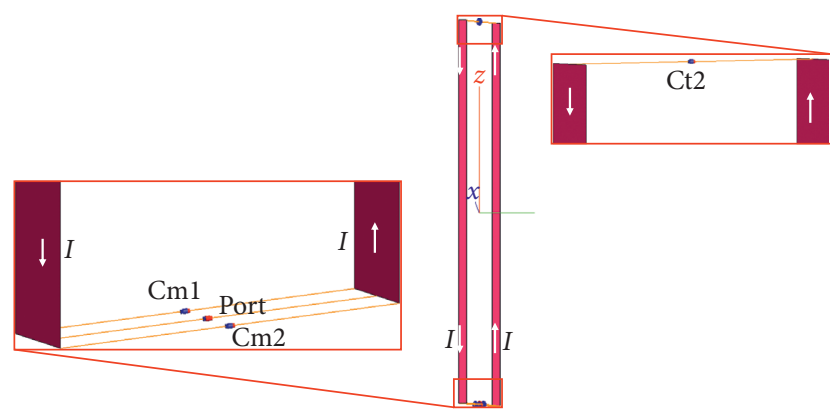

FIgURE 3: Current path in the case of diagonal single-loop (DSL) design.

loaded quality factor $\left(Q_{u_{-} \text {simu }}\right.$ and $\left.Q_{l_{\text {_simu }}}\right)$ values as detailed in the following.

Since an RF coil can be considered an RLC circuit, a theoretical way to estimate the unloaded coil quality factor $Q_{u_{-} \text {theo }}$ is given by [39-41]

$$
Q_{u_{\text {theo }}}=\frac{2 \pi F_{0} L_{\text {coil }}}{R_{\text {coil }}}
$$

where $F_{0}$ is the Larmor frequency, $L_{\text {coil }}$ is the inductance of coil conductors, and $R_{\text {coil }}$ is the estimated coil resistance. Under this condition of the unloaded coil, the losses are represented by the equivalent electrical resistance of the coil $\left(R_{\mathrm{el}}\right)$. The latter can be computed by setting equal both the $Q_{u_{-} \text {theo }}$ equations and the $Q_{u_{-} \text {simu }}$ simulated value, therefore giving [42]

$$
R_{\mathrm{el}}=\frac{2 \pi F_{0} L_{\text {coil }}}{Q_{u_{\text {simu }}}} .
$$

The loop coil inductance was also estimated and derived from Smith abacus. This was done by simulation of the loop at the desired resonance frequency $(63.87 \mathrm{MHz})$ without adding the lumped elements (tuning and matching capacitors). Thus, a first estimation of the electrical resistance of the conductor loop can be derived to check for consistency of the computed $R_{\mathrm{el}}$.

In the case of the loaded coils, simulations were done using a phantom consisting of a cylindrical vessel with a through-hole $(9.2 \mathrm{~mm}$ inner and $45 \mathrm{~mm}$ outer diameters, $50 \mathrm{~mm}$ length) allowing the introduction of each loop coil and filled with a solution of $1.25 \mathrm{~g} \mathrm{NiSO}_{4} \times 6 \mathrm{H}_{2} \mathrm{O}+5 \mathrm{~g} \mathrm{NaCl}$ per liter of distilled water mimicking tissue losses (see Figure 6). The phantom is the dielectric medium that has an electrical propriety of $0.67 \mathrm{~S} / \mathrm{m}$ conductivity, 94.73 relative permittivity/dielectric constant, and $1120 \mathrm{~kg} / \mathrm{m}^{3}$ mass density (http://niremf.ifac.cnr.it/tissprop/).

For all simulations, the phantom was meshed with $2 \mathrm{~mm}$ local mesh size (this was the maximum resolution enabled by FEKO and our hardware). For unloaded loop coils, the mesh was of $0.5 \mathrm{~mm}, 1 \mathrm{~mm}$, and $0.01 \mathrm{~mm}$ triangle edge length, wire segment length, and wire segment radius, respectively. Each loaded loop coil was meshed with a custom mesh of $0.25 \mathrm{~mm}, 0.5 \mathrm{~mm}$, and $0.01 \mathrm{~mm}$ triangle edge length, wire segment length, and wire segment radius, respectively.
Moreover, EM simulations were performed using doubleprecision numerical computations.

Similar to the previous case, a theoretical way to estimate the quality factor $Q_{l_{-} \text {theo }}$ of the loaded coil is given by [40]

$$
Q_{L_{\text {theo }}}=\frac{2 \pi F_{0} L_{\text {coil }}}{R_{\mathrm{el}}+R_{m}}
$$

where $R_{\mathrm{el}}$ is the electrical resistance that represents losses due to the loop coil and $R_{m}$ is the magnetic resistance that represents losses due to the phantom. Under these conditions, $R_{m}$ is given by $[40,42]$

$$
R_{m}=\frac{2 \pi F_{0} L_{\text {coil }}}{Q_{L_{\text {simu }}}}-R_{\mathrm{el}} \text {. }
$$

The total loss resistance $R_{t}$ was then the sum of the electrical resistance of the coil and the magnetic resistance of the phantom and was given by $[17,43]$

$$
R_{t}=R_{\mathrm{el}}+R_{m}
$$

The $\left(B_{1 x, y} / I \sqrt{R_{t}}\right)$ term (which is proportional to the SNR distribution) of each loop geometry was then calculated. They were analyzed in regions close to the coil, which corresponds to the colon wall location. By using Matlab software, the intensity values and radial uniformity of signal distribution were assessed in a region defined by a disc centered on the coil axis and having $5 \mathrm{~mm}$ and $15 \mathrm{~mm}$ inner and outer radiuses (the targeted colon wall imaging area). To achieve that $\left(B_{1 x, y} / I \sqrt{R_{t}}\right)$ values were calculated on concentric circles (with $10^{\circ}$ angle sampling from $0^{\circ}$ to $360^{\circ}$ ), for each chosen distance and specific coil orientation with respect to $B_{0}$. As already mentioned, the studied orientations correspond to the loop coil rotating around the $x$-axis with an angle $\alpha=0^{\circ}, 45^{\circ}$, and $90^{\circ}$. From the sampled values, means and standard deviations were calculated and the subsequent coefficient of variation $(\mathrm{CV}=$ standard deviation/mean) was derived.

Finally, the geometries providing the best signal uniformity (measured through the $\mathrm{CV}$ ) at different coil orientations and for different imaging distances were compared. They were then combined in a theoretical single reconfigurable endoluminal coil using MEMS switches [22]. Figure 7 summarizes the different steps from the design of the loop geometry until the analysis of the results.

Tuning and matching capacitor values of each unloaded or/and loaded loop configuration are summarized in Table 1.

\section{Results}

To evaluate the performance of the different loop geometries presented in the Method section, we ran the comparisons in two steps. First, all configurations of double loops were evaluated to determine the one that would be best suited for endoluminal colon imaging. In a second step, the chosen double-loop configurations were compared with the diagonal single loop (DSL) since it is the state-of-the-art reference solution. 


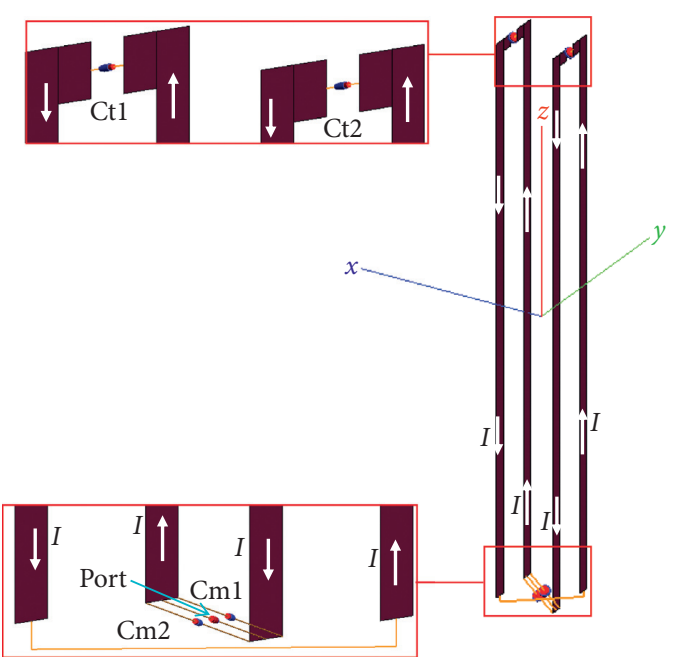

(a)

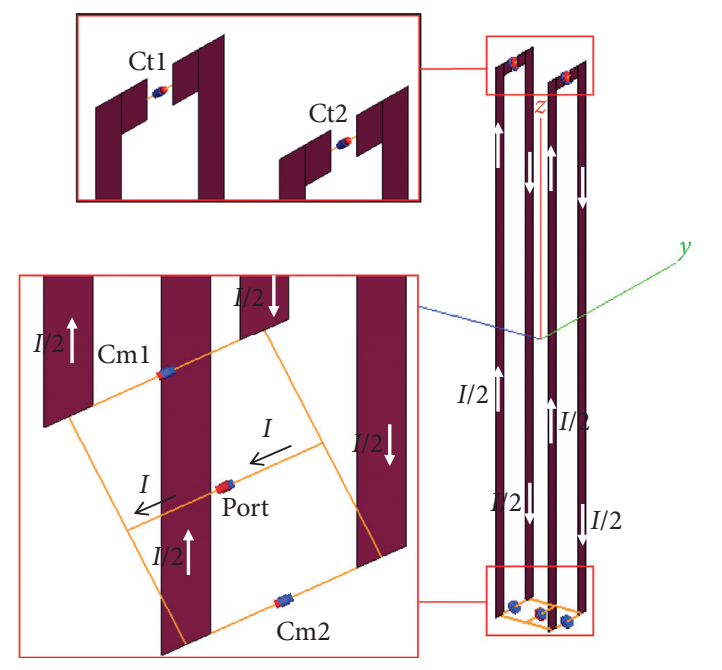

(c)

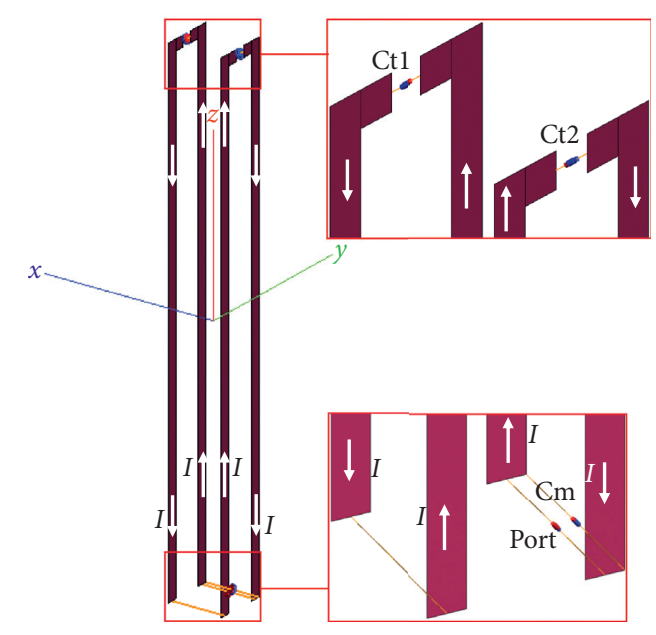

(b)

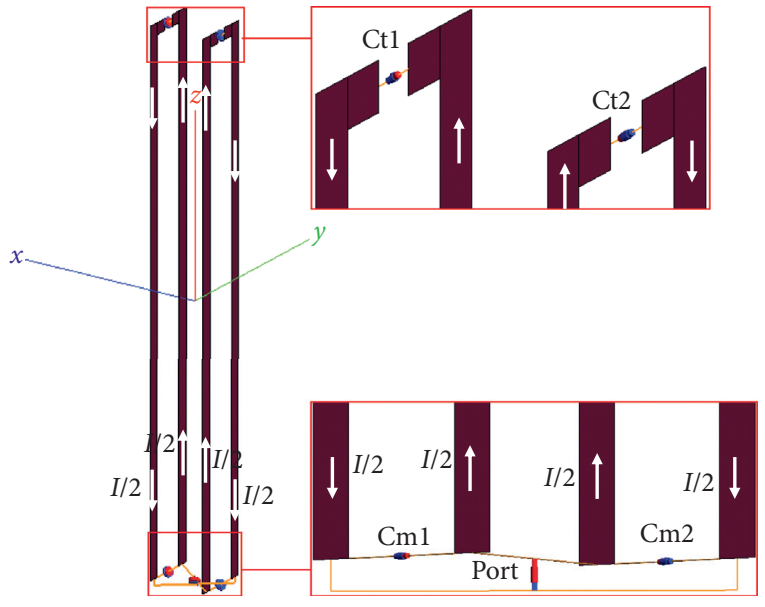

(d)

FIgURE 4: Current path possibilities in the case of rectangular double-loop (RDL) designs. Rectangular (a) double-layer (i.e., loops are in parallel and thus ( I/2) current flows in each one) and (b) double-turn (i.e., loops are in series and thus $I$ current flows in each one) coils are designed to obtain currents circulating in the same directions (RDL-SC and RDT-SC). Also, rectangular (c) double-layer and (d) doubleturn coils are designed to obtain currents circulating in the opposite directions (RDL-OC and RDT-OC).

\subsection{Part I: Unloaded Rectangular and Diagonal Double-Layer/} Double-Turn Loops. Since the simulated values of $R_{m}$ for the double-layer/double-turn loops are much smaller than $R_{\mathrm{el}}$, only results of unloaded coils will be presented in this section, and unloaded and loaded simulations will lead to almost the same conclusions. Because SNR is proportional to the $\left(B_{1 x, y} / I \sqrt{R_{t}}\right)$ ratio, the results of $\left(B_{1 x, y} / I \sqrt{R_{t}}\right)$ values of rectangular and diagonal double loops, sampled every $10^{\circ}$ on circles of $10 \mathrm{~mm}$ radius (centered on the loop center), are displayed in Figure 8 . As can be seen, configurations that display higher $\left(B_{1 x, y} / I \sqrt{R_{t}}\right)$ intensity also tend to have higher variations with respect to the sampling angle. In addition, the corresponding $\mathrm{CVs}$ ( $\mathrm{CV}=$ standard deviation/ mean) for the different geometries and in the reference orientation $\left(0^{\circ}\right)$ are displayed in Figure 9. The RDL-OC and RDT-OC offer smaller CVs than the DDL or DDT; therefore, RDL-OC and RDT-OC are the two geometries that will be considered for the rest of this work ( $\mathrm{CV}$ criterion is more important in the compromise case).

3.2. Part II: Loaded Single and Double Loops. These two selected geometries (RDL-OC and RDT-OC) were compared with the diagonal single loop (DSL) and for the orientations of the loops about $x$-axis of $0^{\circ}, 45^{\circ}, 90^{\circ}$ and at different distances $(5-15 \mathrm{~mm})$ from each loop center. This time, all simulations were run in loaded conditions since the simulated $R_{m}$ values were quite different between the DSL and double-loop geometries.

3.2.1. Mean of $\left(B_{1 x, y} / I \sqrt{R_{t}}\right)$ versus Distance at Specific $R F$ Loop Rotation. As shown in Figure 10, the mean signal intensity (mean $\left.\left(B_{1 x, y} / I \sqrt{R_{t}}\right)\right)$ values for all loops dropped off rapidly when moving away from the loop center. The DSL 


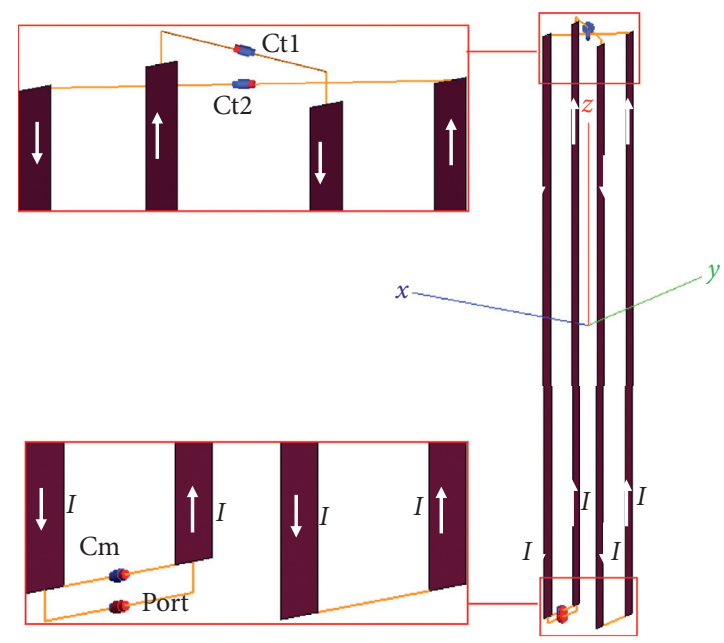

(a)

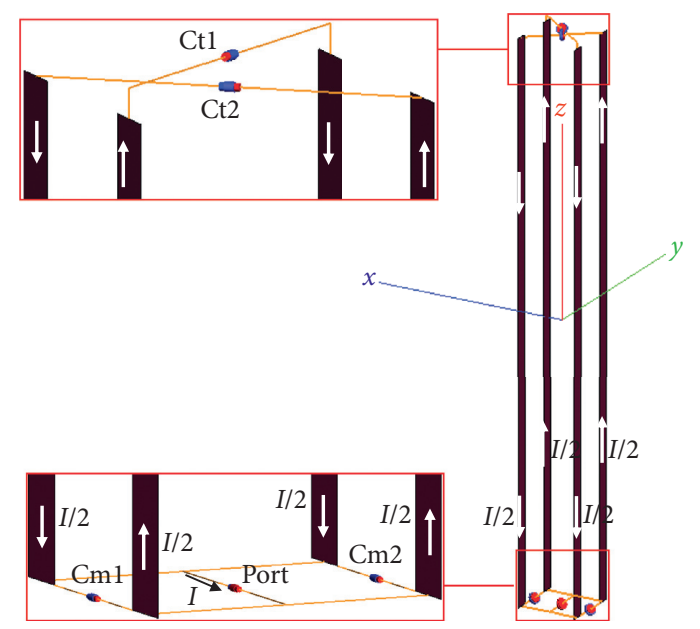

(c)

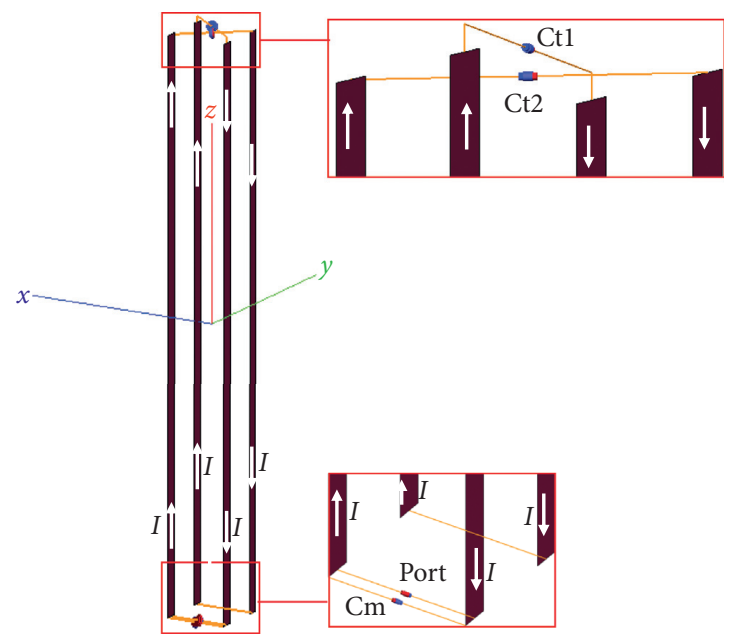

(b)

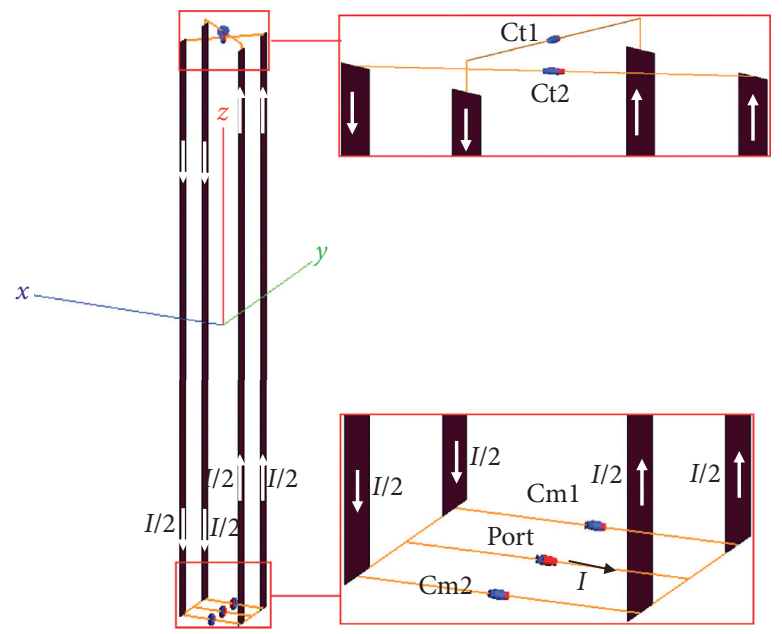

(d)

FIGURE 5: Current path possibilities in the case of diagonal double-loop (DDL) designs. Diagonal (a) double-layer (i.e., loops are in parallel and thus ( $I / 2)$ current flows in each one) and (b) double-turn (i.e., loops are in series and thus $I$ current flows in each one) coils are designed to obtain currents circulating in direction D1 (DDL-D1 and DDT-D1). Also, diagonal (c) double-layer and (d) double-turn coils are designed to obtain currents circulating in direction D2 (DDL-D2 and DDT-D2).

presented mean $\left(B_{1 x, y} / I \sqrt{R_{t}}\right)$ values higher than the other loops for all distances and loop orientations. The mean $\left(B_{1 x, y} / I \sqrt{R_{t}}\right)$ values obtained for RDL-OC and RDT-OC displayed nearly identical mean values for all distances and for the three orientations considered. However, their mean values were also inferior to those of the DSL in all test conditions.

3.2.2. Coefficients of Variation (CV) of $\left(B_{1 x, y} / I \sqrt{R_{t}}\right)$ versus Distance at Specific RF Loop Rotation. Coefficients of variation for the three geometries and for distances between 5 and $15 \mathrm{~mm}$ are plotted in Figures 11(a)-11(c) corresponding to the three orientations investigated $\left(0^{\circ}, 45^{\circ}\right.$, and $\left.90^{\circ}\right)$.

At the reference orientation (see Figure 11(a)) and for distances inferior to $9 \mathrm{~mm}$, the CV values of RDL-OC and RDT-OC were very close to each other and smaller than those displayed by the DSL. Between 9 and $12 \mathrm{~mm}$, the DSL is the geometry displaying the smallest $\mathrm{CV}$, but above $12 \mathrm{~mm}$ it is the RDT-OC that displays the smallest CV.
At $45^{\circ}$ loop orientation (see Figure 11(b)), the RDT-OC and RDL-OC geometries show the smallest CV until $8 \mathrm{~mm}$, but above $8 \mathrm{~mm}$, the DSL is the best suited geometry.

By further increasing the loop rotation angle and in particular at the $90^{\circ}$ position (see Figure $11(\mathrm{c})$ ), the DSL exhibits the smallest CV for all distances.

\section{Discussion}

In our application, the coil is placed very close to the region of interest. This leads to very high signal intensities close to the coil conductors. However, there is a rapid signal decrease when moving away from the coil center, as can be observed in all surface coils [20]. Starting from a given coil geometry with given positions of conductors, multiple single-channel double-turn/double-layer models were designed and compared with a diagonal single-loop geometry that will serve as reference. In this study, we focused on the combination of two main criteria: $\left(B_{1 x, y} / I \sqrt{R_{t}}\right)$ signal intensity and 


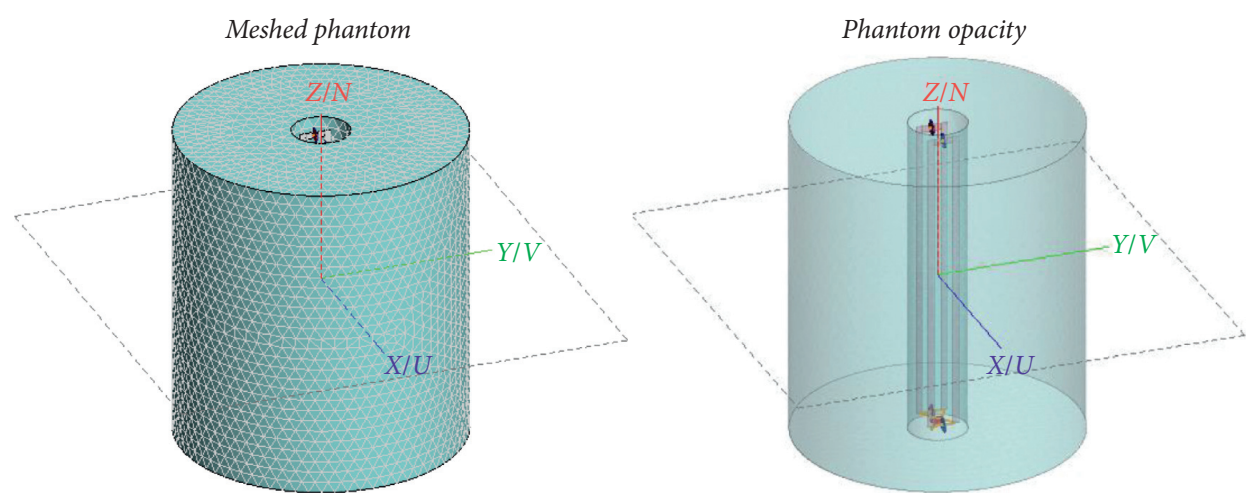

Figure 6: The phantom used for simulation of different loaded loop coils. It consists of a cylindrical vessel with a through-hole with dimensions of $9.2 \mathrm{~mm}$ inner and $45 \mathrm{~mm}$ outer diameters, and $50 \mathrm{~mm}$ length, and is filled with a solution of $1.25 \mathrm{~g} \mathrm{NiSO} 4 x 6 \mathrm{H}_{2} \mathrm{O}+5 \mathrm{~g} \mathrm{NaCl}$ per liter of distilled water mimicking tissue losses $\left(0.67 \mathrm{~S} / \mathrm{m}\right.$ conductivity, 94.73 relative permittivity/dielectric constant, and $1120 \mathrm{~kg} / \mathrm{m}^{3}$ mass density). It was meshed with a $2 \mathrm{~mm}$ local mesh size.

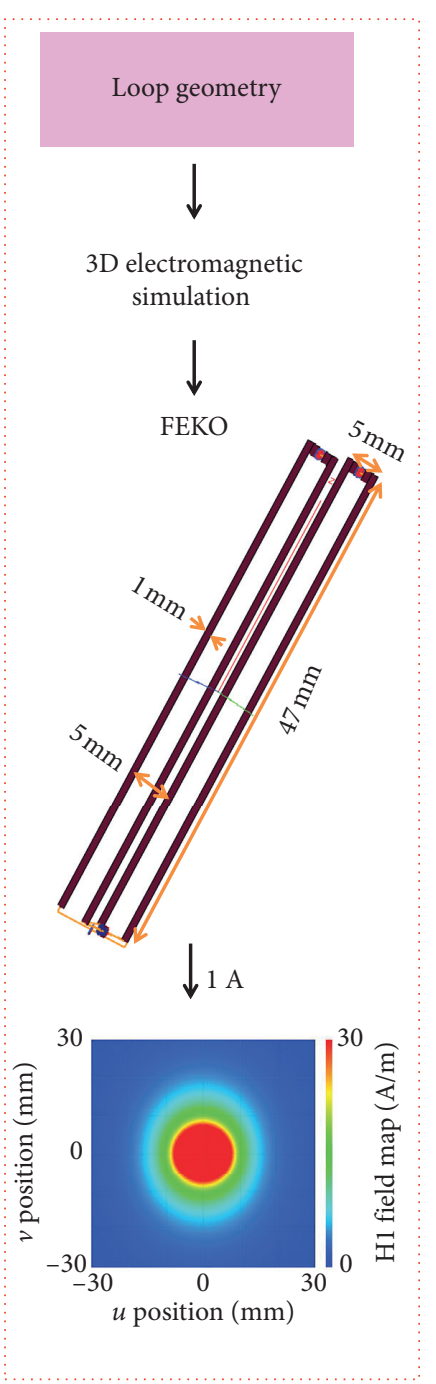

(a)

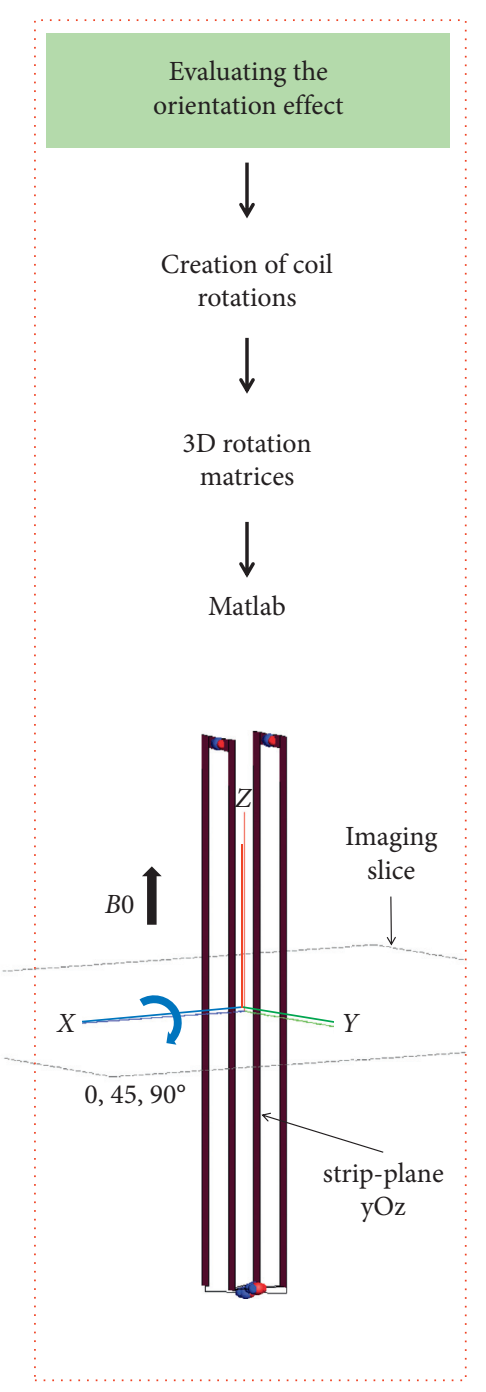

(b)

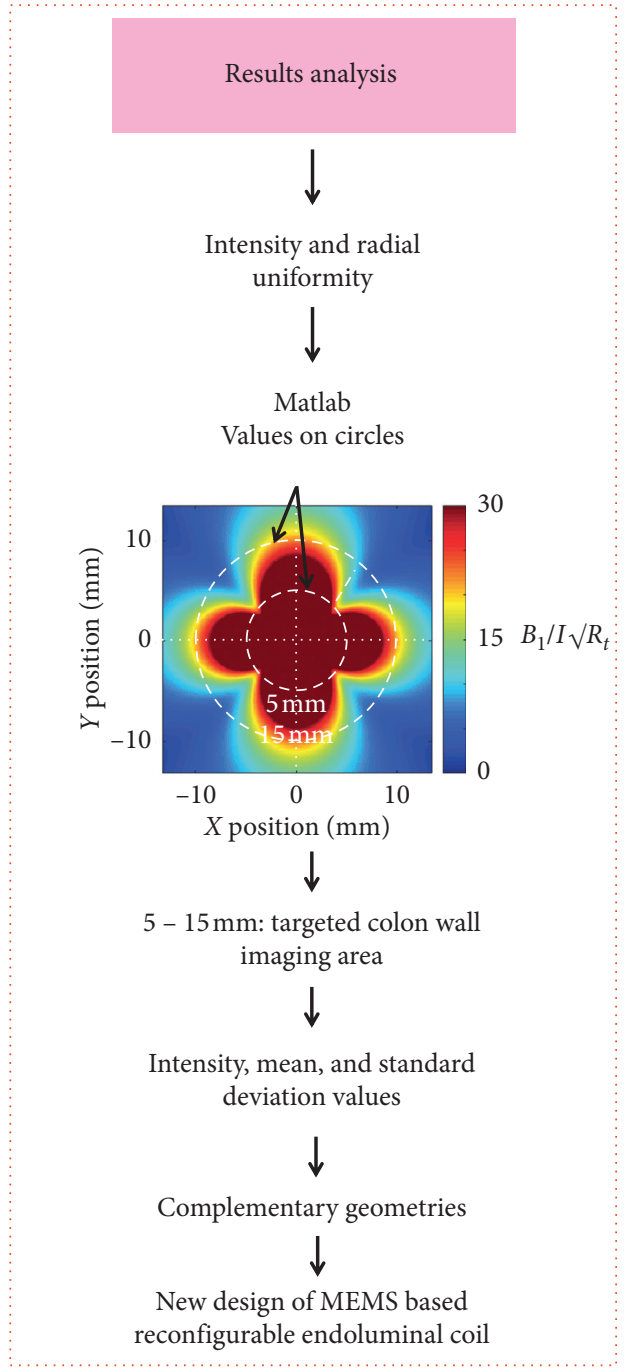

(c)

FIGURE 7: Different steps from the coil design to the analysis of results. (a) Full-wave electromagnetic simulation with FEKO software to get the magnetic field H1. (b) Application of the coil-orientation effect about $x$-axis on $\left(B_{l x, y} / I \sqrt{R_{t}}\right)$ (which is proportional to the SNR) distribution by using Matlab software (3D transformation based on rotation matrices). (c) Analysis of the results corresponding to the chosen criteria: coefficient of variation between $5 \mathrm{~mm}$ and $15 \mathrm{~mm}$ from the center of the coil (targeted colon wall imaging area). 
TABLE 1: Tuning and matching capacitor values of each unloaded or/and loaded loop configuration.

\begin{tabular}{|c|c|c|c|c|}
\hline \multirow[t]{2}{*}{ Loop geometry } & \multicolumn{2}{|c|}{$\begin{array}{l}\text { Tuning capacitor } \\
\qquad(\mathrm{pF})\end{array}$} & \multicolumn{2}{|c|}{$\begin{array}{c}\text { Matching } \\
\text { capacitor }(\mathrm{pF})\end{array}$} \\
\hline & $C_{\mathrm{t} 1}$ & $C_{\mathrm{t} 2}$ & $C_{\mathrm{m} 1}$ & $C_{\mathrm{m} 2}$ \\
\hline \multicolumn{5}{|c|}{ Unloaded rectangular double-loop (RDL) coils } \\
\hline RDL-SC & 124.88 & 124.88 & 447.31 & 447.31 \\
\hline RDT-SC & 104.62 & 104.62 & 229.71 & 229.71 \\
\hline RDL-OC & 157.19 & 157.19 & 424.34 & 424.34 \\
\hline RDT-OC & 127.00 & 127.00 & 414.98 & - \\
\hline \multicolumn{5}{|c|}{ Unloaded diagonal double-loop (DDL) coils } \\
\hline DDL-D1 & 113.75 & 113.75 & 425.00 & 425.00 \\
\hline DDT-D1 & 99.36 & 99.36 & 447.78 & - \\
\hline DDL-D2 & 94.95 & 94.95 & 410.00 & 410.00 \\
\hline DDT-D2 & 84.75 & 84.75 & 414.18 & - \\
\hline \multicolumn{5}{|c|}{ Loaded single and double-loop coils } \\
\hline DSL & - & 96.5 & 303.07 & 303.07 \\
\hline RDL-OC & 157.19 & 157.19 & 424.34 & 424.34 \\
\hline RDT-OC & 127.00 & 127.00 & 414.98 & - \\
\hline
\end{tabular}

variation characterized by the mean and standard deviations measured on concentric circles centered on the coil center. These two measures were combined to obtain CVs that will reflect signal uniformity in the regions of interest. It is difficult to define threshold values of acceptable CVs but it is still possible to define which geometry fares better for the different orientations $\left(0^{\circ}, 45^{\circ}\right.$, and $\left.90^{\circ}\right)$.

In the first step, the simulation results of different configurations of the rectangular and diagonal double loops led to the selection of a rectangular double layer and double turn with opposite current directions (RDL-OC and RDT$\mathrm{OC})$ since they were the ones that displayed the smallest CVs.

In a second step, the simulation results of the different loaded single- and double-loop coils were studied for distances ranging between $5 \mathrm{~mm}$ and $15 \mathrm{~mm}$ and for three specific orientations. As mentioned in Materials and Methods section, the choice of the three orientations was made according to the plausible orientation of the coil during navigation in the colon during an MR examination. In the different vertical regions (rectum, descending colon, and ascending colon), we assumed that each coil is oriented at $0^{\circ}$ with respect to the $x$-axis. In this case, the RDL-OC and RDT-OC presented the best signal uniformity at low distances $(<9 \mathrm{~mm})$. For distances between 9 and $12 \mathrm{~mm}$, the DSL exhibits higher signal intensity and smaller CVs. Above $12 \mathrm{~mm}$, the RDT-OC exhibits smaller CVs.

In the horizontal region (middle part of the transverse colon), we assumed that each coil has operated at $90^{\circ}$ rotation around the $x$-axis. The DSL presents the best signal uniformity for all distances considered. This is understandable since for this particular orientation, the RDL-OC and RDT-OC both have their coil planes perpendicular to $B_{0}$ (which is the worst-case scenario for signal uniformity), whereas the DSL, due to its particular plane orientation, has its normal to the coil plane at $45^{\circ}$ to $\mathrm{B}_{0}$. Thus, the DSL appears to be the best solution in this case. It should be noted that rotating the DSL by a further $45^{\circ}$, but this time around the $y$-axis, would enable an even better signal uniformity since the coil plane would then lie in the horizontal plane of the MR scanner. However, this is not within the scope of this work that considers reconfiguration and not reorientation of coils.

In the tilted regions (sigmoid and the two extremities of the transverse colon), we assumed that each coil was oriented at $45^{\circ}$ with respect to the $x$-axis. In this case, the RDLOC and RDT-OC present the best uniformity up to $8 \mathrm{~mm}$, and the DSL does so for higher distances $(>8 \mathrm{~mm})$.

To summarize, after the analysis of these simulated results given by every individual geometry, the selection of complementary loop geometries emerged as a relevant possibility to provide improved robustness regarding the coil orientations. It is now necessary to see how it would be possible to switch between these three geometries using MEMS.

4.1. Proposed Design of the MEMS-Based Reconfigurable Endoluminal Coil. The proposed MEMS-based reconfigurable endoluminal coil (swiM RE-Coil) design is illustrated in Figure 12. It consists of using only four copper strips (defined in $u O n$-plane) and two electronic units (see Figure 12(a)). The latter include sets of capacitors and MEMS switches located at the proximal and distal parts of the loop (Figure 12(b)).

It is possible to use the RDT-OC, but it requires the use of more components (MEMS and capacitors). For this reason and given that at the reference orientation $\left(0^{\circ}\right)$, the $\mathrm{CV}$ of the RDT-OC is only marginally better than that of the DSL above $12 \mathrm{~mm}$ in the proposed design; only the RDL-OC and DSL were considered, such that the final design includes the following:

(i) At $0^{\circ}$ : RDL-OC below $9 \mathrm{~mm}$ and DSL above $9 \mathrm{~mm}$

(ii) At $45^{\circ}$ : RDL-OC below $8 \mathrm{~mm}$ and DSL above $8 \mathrm{~mm}$

(iii) At $90^{\circ}$ : DSL

The active decoupling state is ensured by opening all MEMS switches: S1 to S4 (the MEMS switch can ensure a high isolation: $10 \mathrm{M} \Omega$ ). This ensures that there is no closed loop in the design (see Figure 12(c)). The DSL loop geometry is obtained by closing S1 and S2 and opening all other switches (see Figure 12(e)), and it is used at high distances for vertical and tilted regions and in all conditions for horizontal regions. Finally, the RDL-OC is obtained by closing S3 and S4 and opening all other switches (see Figure 12(f)), and it is used at low distances for tilted and vertical regions.

Although different and independent encapsulated coil loops could be considered, this work focuses on the potential use of MEMS in series in order to use the capability offered to reconfigure the loop path and geometry.

As previously demonstrated in the literature [23], MEMS switches allow one to alter the loop geometry while being connected to a unique receiver channel. The present simulation work was performed by keeping in mind this overall objective in order to use this feature when coil orientation has to change with location within the colon. Indeed, 


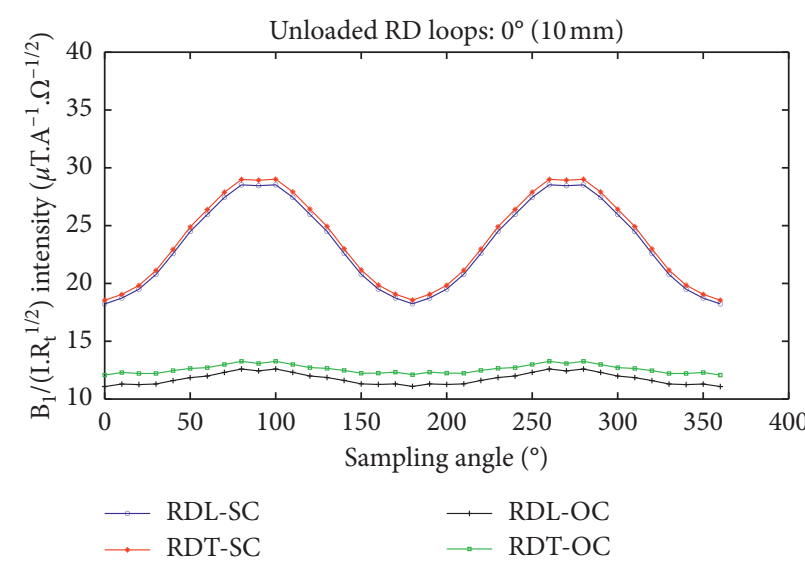

(a)

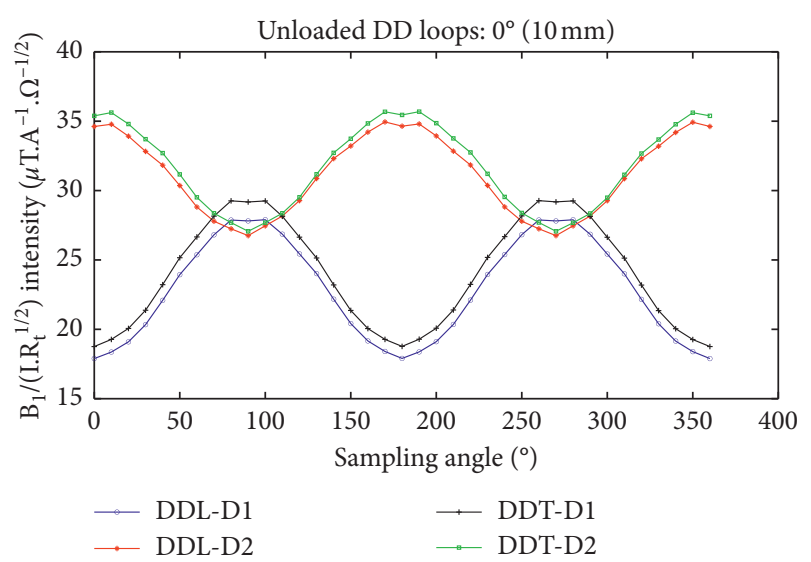

(b)

Figure 8: Unloaded $\left(B_{l x, y} / I \sqrt{R_{t}}\right)$ intensity versus sampling angle for (a) rectangular double-loop and (b) diagonal double-loop coils at reference orientation and at $10 \mathrm{~mm}$ distance from the loop center at reference orientation $\left(0^{\circ}\right)$.

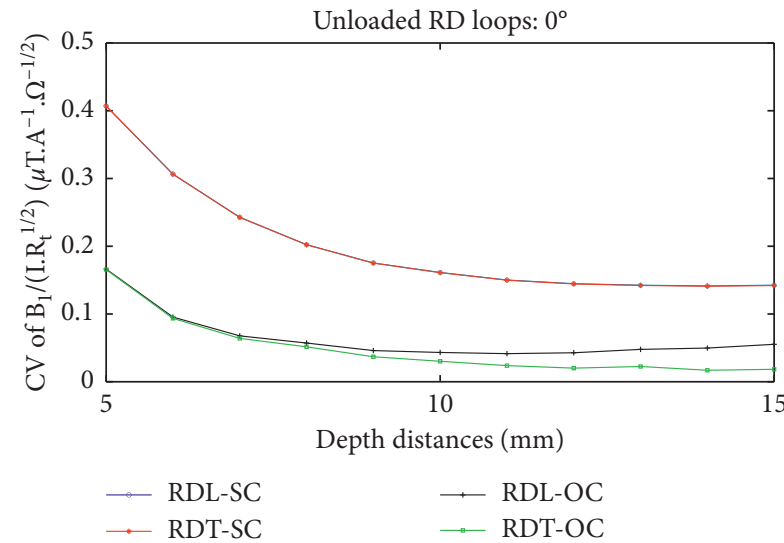

(a)

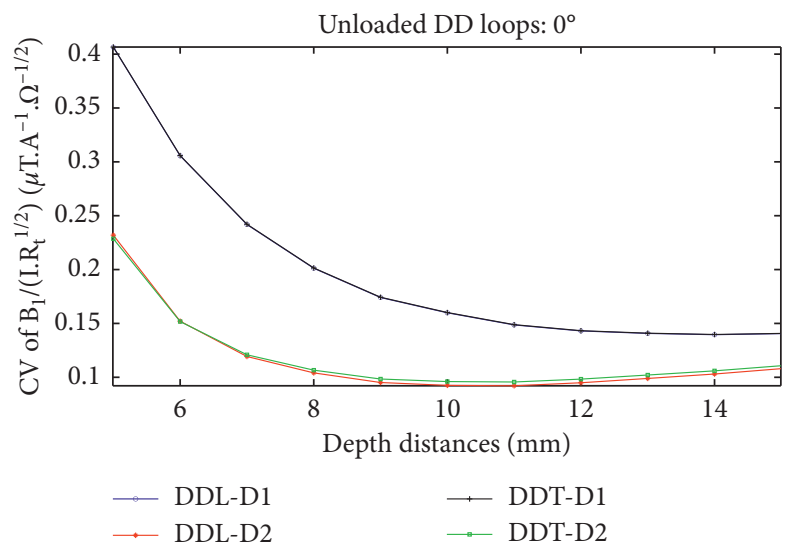

(b)

Figure 9: The CVs (coefficients of variation) versus distance of unloaded (a) rectangular double (RD) loops and (b) diagonal double (DD) loops with loops in the reference orientation $\left(0^{\circ}\right)$.

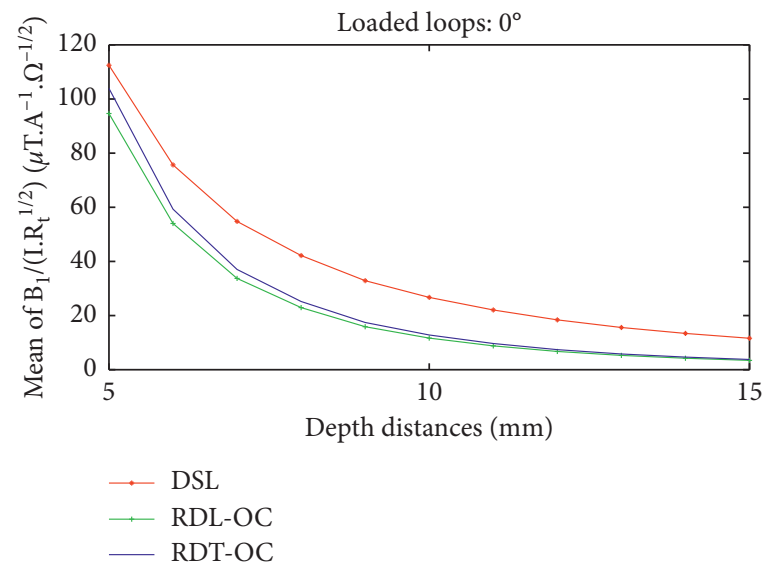

(a)

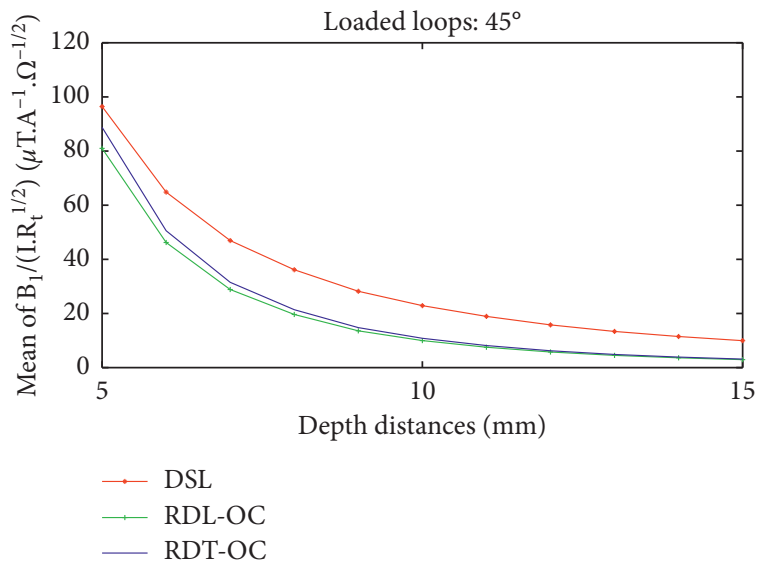

(b)

Figure 10: Continued. 


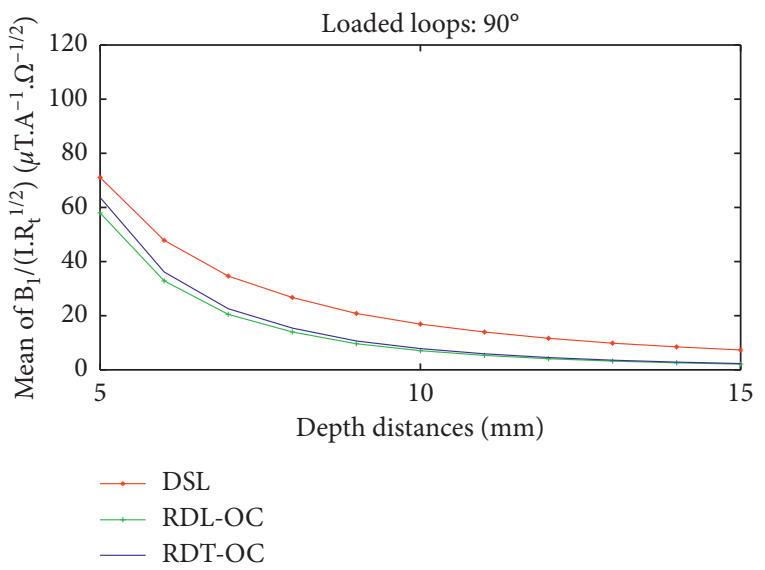

(c)

Figure 10: Means of $\left(B_{l x, y} / I \sqrt{R_{t}}\right)\left(B_{l x, y} / I \sqrt{R_{t}}\right)$ ratio (calculated on concentric circles) versus distance from each loop center (from 5 to $15 \mathrm{~mm}$ ) at specific orientations with respect to the $x$-axis: angles of (a) $0^{\circ}$, (b) $45^{\circ}$, and (c) $90^{\circ}$.

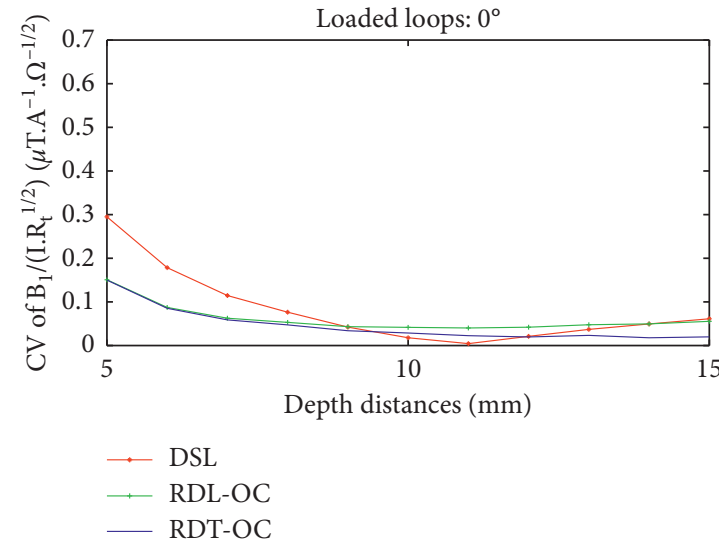

(a)

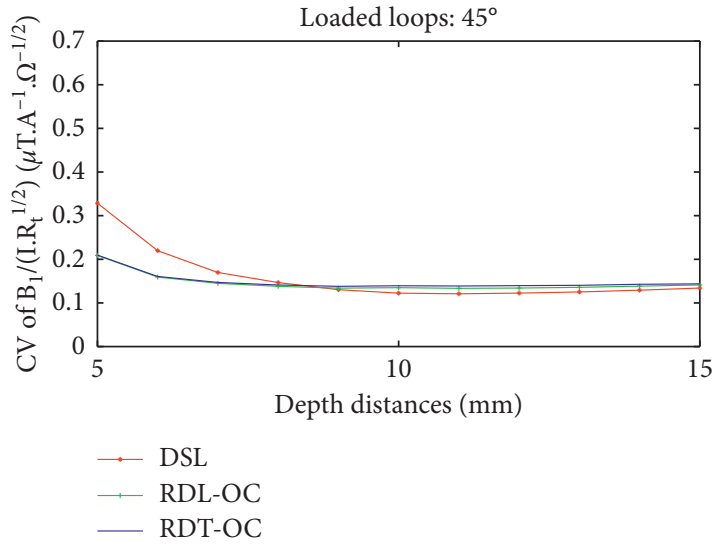

(b)

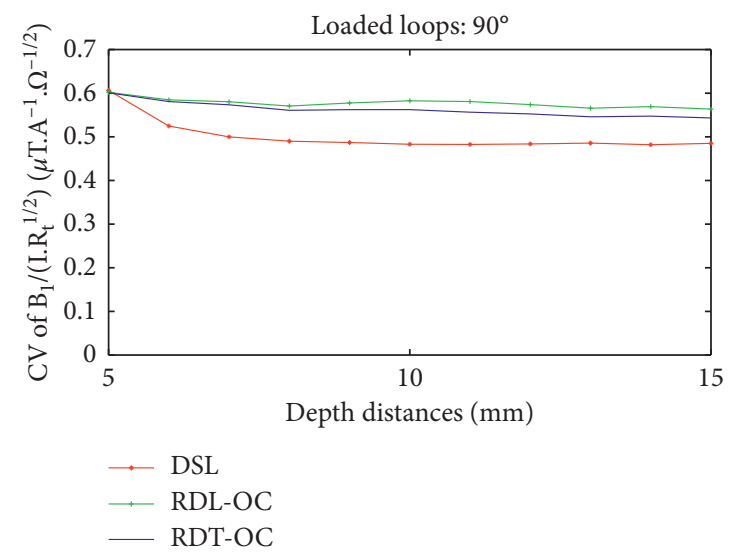

(c)

Figure 11: Coefficients of variation (CV) of $\left(B_{l x, y} / I \sqrt{R_{t}}\right)$ ratio (calculated on concentric circles) versus distance from each loop center (from 5 to $15 \mathrm{~mm}$ ) at specific orientations with respect to the $x$-axis: angles of (a) $0^{\circ}$, (b) $45^{\circ}$, and (c) $90^{\circ}$.

comparing theoretical nonreconfigurable and reconfigurable designs would clearly be at the advantage of nonreconfigurable coils. Moreover, to place the MEMS in series with the loop coil adds an additional resistance. As shown in a previous work [29] comparing coil prototypes with a reference coil using a PIN diode in parallel with MEMS placed in series, the quality factor was reduced from 62 to 41 . It is, however, not possible to strictly and even theoretically 

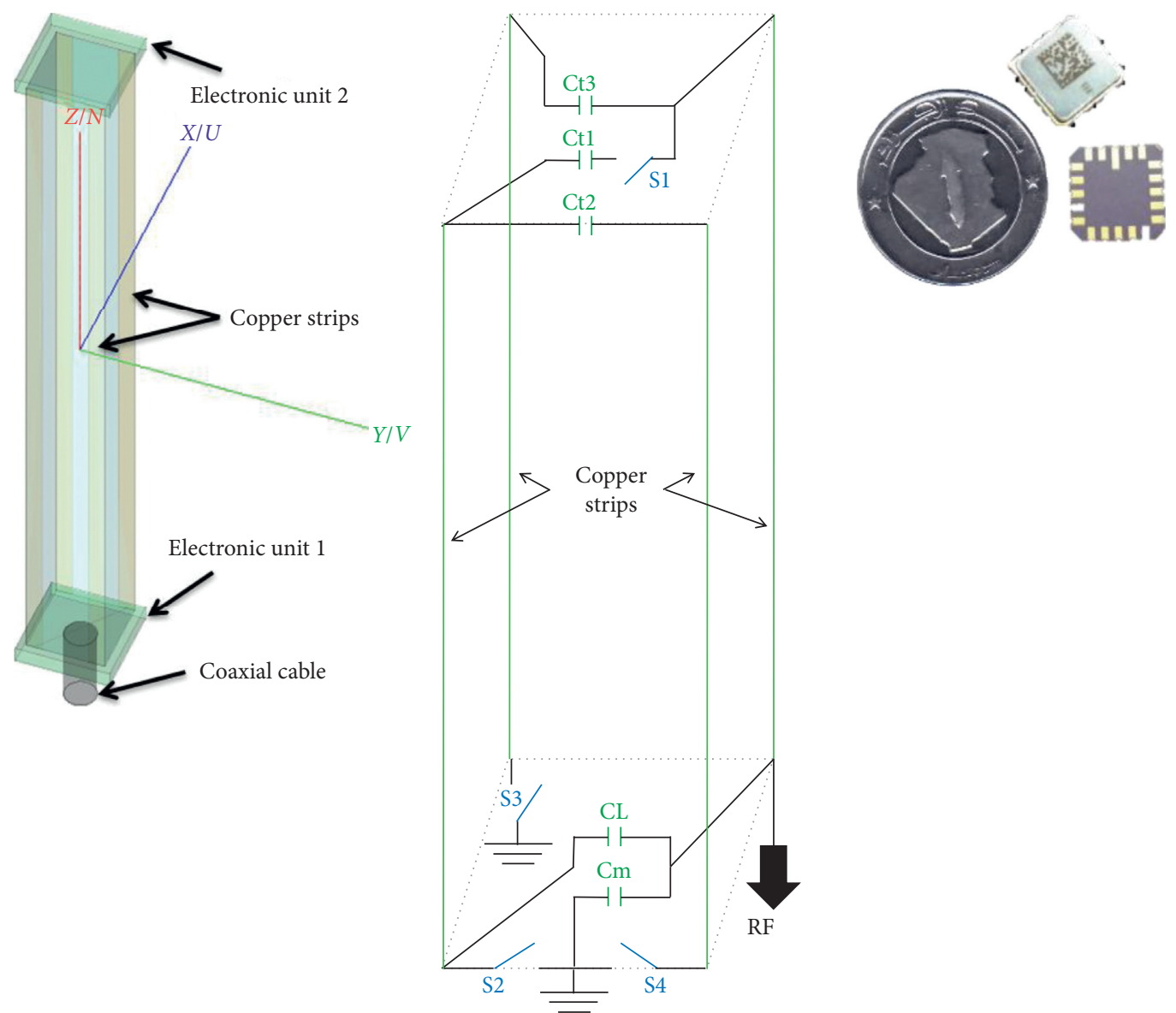

(a)

(b)

(c)

FIgURE 12: Continued. 


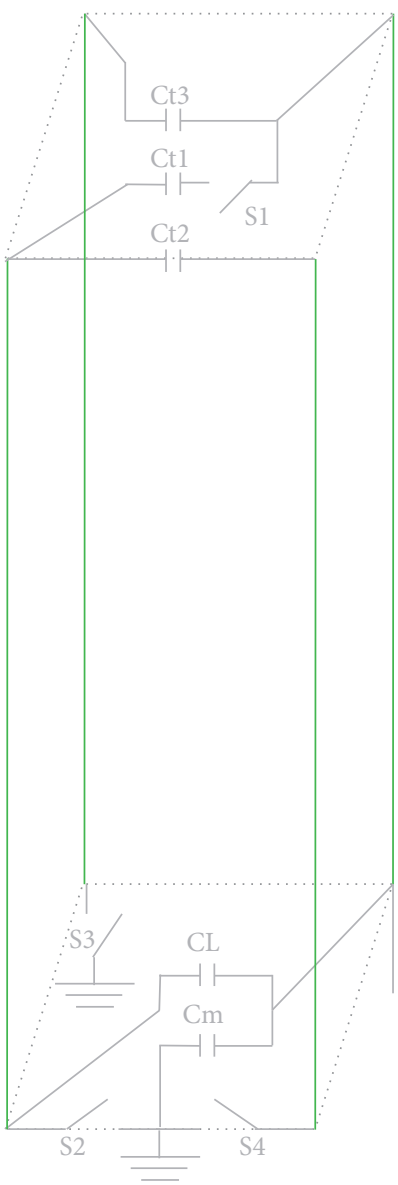

(d)

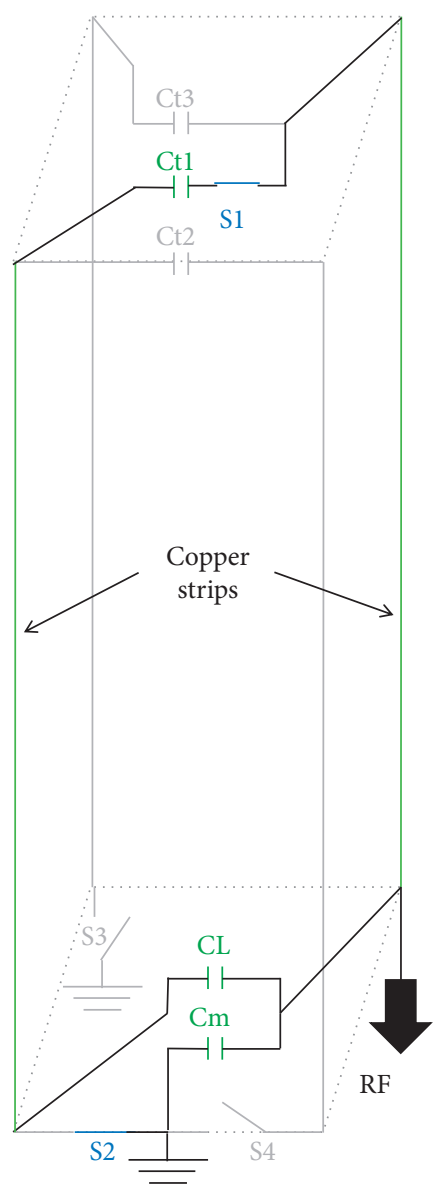

(e)

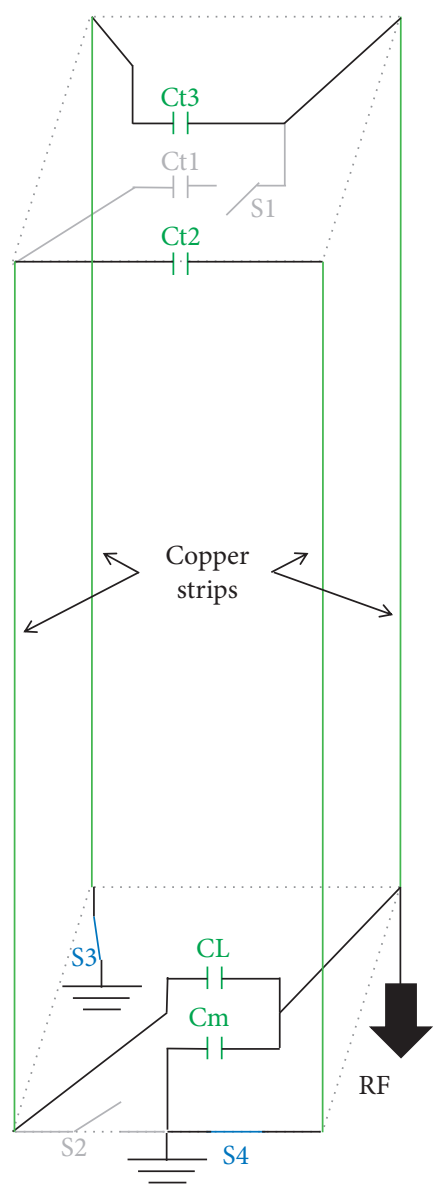

(f)

Figure 12: (a) The proposed and (b) detailed MEMS-based reconfigurable endoluminal coil (swiM RE-Coil) architecture solution, which consists of four copper strips, four switches, three tuning capacitors, and two matching capacitors. (c) An example of MR switches, MEMS switch, which we propose and advice to use for this design. (d) The active decoupling state. (e) The DSL loop geometry formed by closing S1 and S2 switches and opening S3 and S4. (f) The RDL-OC geometry formed by opening S1 and S2 switches and closing S3 and S4.

consider similar comparisons because MEMS allow for the use of a common conductor, which cannot be done when using, for instance, two independent coils. Moreover, due to the close proximity of the geometries, the effect of mutual coupling would be difficult to handle and the impact on the SNR distribution difficult to estimate.

Another solution reported in the literature (the commercial Noras 2 ch endoanal coils) is based on the use of two rectangular perpendicular loops with two channels. It may be optimal in the case of multiple receive channels to perform a geometrical decoupling and improve the SNR by a $\sqrt{ } 2$ factor with an improvement in the signal distribution. In our signal-channel case, geometrical decoupling is not an issue, but distribution is penalized because of the added resistive losses.

\section{Conclusion}

In conclusion, endoluminal MRI is currently the best alternative for achieving the required SNR per unit time, enabling proper visualization of the colon wall layers. However, the signal distribution (intensity and uniformity of the coil sensitivity) is affected during coil navigation within the colon since it leads to a modification in the coil orientation with respect to the static magnetic field. Reconfiguration of loop coil geometry according to the colon area (coil orientation) and the distance from the coil center is an interesting way to reduce this dependency and thus improve the coil sensitivity uniformity and image quality. To achieve the switch between loop configurations, the use of MEMS switches is an attractive solution but in practice it remains a challenge in the case of endoluminal imaging where available space and component sizes are critical. The proposed design of a reconfigurable endoluminal coil is based on four MEMS switches and makes it possible not only to switch between RDL-OC and DSL but also to achieve an accurate active decoupling of the endoluminal coil during the RF transmission of an MR experiment.

\section{Data Availability}

No data were used to support this study.

\section{Conflicts of Interest}

The authors declare that they have no conflicts of interest. 


\section{Acknowledgments}

The authors would like to thank GE Healthcare, Inc., and ANRT for funding this project (CIFRE no. 2016/0486). This work was conducted within the scope of the Labex PRIMES of the University of Lyon (ANR-11-LABX-0063), in the program "Investissements d'Avenir" (ANR-11-IDEX-0007), run by the French National Research Agency (ANR).

\section{References}

[1] R. W. Geenen, S. M. Hussain, and P. D. Siersema, "Current status of MRI in patients with inflammatory bowel disease colitis," Applied Radiology, vol. 36, no. 2, p. 10, 2007.

[2] P. Munkholm, "Review article: the incidence and prevalence of colorectal cancer in inflammatory bowel disease," Alimentary Pharmacology \& Therapeutics, vol. 18, pp. 1-5, 2003.

[3] J. Ferlay, M. Colombet, I. Soerjomataram et al., "Estimating the global cancer incidence and mortality in 2018: GLOBOCAN sources and methods," International Journal of Cancer, vol. 144, no. 8, pp. 1941-1953, 2019.

[4] X. V. Qadir, M. Clyne, T. K. Lam, M. J. Khoury, and S. D. Schully, "Trends in published meta-analyses in cancer research, 2008-2013," Cancer Causes \& Control, vol. 28, no. 1, pp. 5-12, 2017.

[5] L. A. Torre, F. Bray, and R. L. Siegel, "Global cancer statistics, 2012," CA: A Cancer Journal for Clinicians, vol. 65, no. 2, pp. 87-108, 2015.

[6] C. Joachim, J. Véronique-Baudin, M. Razanakaivo et al., "Trends in colorectal cancer in the Caribbean: a populationbased study in Martinique, 1982-2011," Revue d'Épidémiologie et de Santé Publique, vol. 65, no. 3, pp. 181-188, 2017.

[7] J. B. O’Connell, M. A. Maggard, and C. Y. Ko, "Colon cancer survival rates with the new american joint committee on cancer sixth edition staging," JNCI Journal of the National Cancer Institute, vol. 96, no. 19, pp. 1420-1425, 2004.

[8] A. Laader, K. Beiderwellen, O. Kraff et al., "1.5 versus 3 versus 7 Tesla in abdominal MRI: a comparative study," PLoS One, vol. 12, no. 11, Article ID e0187528, 2017.

[9] M. Maas, D. M. J. Lambregts, M. J. Lahaye et al., “T-staging of rectal cancer: accuracy of 3.0 Tesla MRI compared with 1.5 Tesla," Abdominal Imaging, vol. 37, no. 3, pp. 475-481, 2012.

[10] T. Akasu, G. Iinuma, T. Fujita et al., "Thin-section MRI with a phased-array coil for preoperative evaluation of pelvic anatomy and tumor extent in patients with rectal cancer," American Journal of Roentgenology, vol. 184, no. 2, pp. 531538, 2005.

[11] E. Van Cutsem, H. Verheul, P. Flamen et al., "Imaging in colorectal cancer: progress and challenges for the clinicians," Cancers, vol. 8, no. 9, p. 81, 2016.

[12] G. J. Metzger, P.-F. van de Moortele, C. Akgun et al., "Performance of external and internal coil configurations for prostate investigations at $7 \mathrm{~T}$," Magnetic Resonance in Medicine, vol. 64, no. 6, pp. 1625-1639, 2010.

[13] S. Giusti, P. Buccianti, M. Castagna et al., "Preoperative rectal cancer staging with phased-array MR," Radiation Oncology, vol. 7, no. 1, p. 29, 2012.

[14] G. C. Hurst, J. Hua, J. L. Duerk, and A. M. Cohen, "Intravascular (catheter) NMR receiver probe: preliminary design analysis and application to canine iliofemoral imaging," Magnetic Resonance in Medicine, vol. 24, no. 2, pp. 343-357, 1992.
[15] D. J. Gilderdale, N. M. DeSouza, G. A. Coutts et al., "Design and use of internal receiver coils for magnetic resonance imaging," The British Journal of Radiology, vol. 72, no. 864, pp. 1141-1151, 1999.

[16] H. Dorez, R. Sablong, L. Canaple et al., "Endoluminal highresolution MR imaging protocol for colon walls analysis in a mouse model of colitis," Magnetic Resonance Materials in Physics, Biology and Medicine, vol. 29, no. 4, pp. 657-669, 2016.

[17] J.-M. Verret, F. Pilleul, C. Rabrait et al., "Characterization of a dedicated double loop, endoluminal coil for anal sphincter MR imaging at 1.5 T and 3 T," Concepts in Magnetic Resonance Part B: Magnetic Resonance Engineering, vol. 44, no. 2, pp. 39-49, 2014.

[18] S. A. Winkler, I. Saniour, and A. Chaudhari, "MRSaiFE: tissue heating prediction for MRI: a feasibility study," in 2020 IEEE MTT-S International Microwave Biomedical Conference (IMBioC), pp. 1-3, IEEE, Toulouse, France, December 2020.

[19] R. Ayde, G. Gaborit, P. Jarrige et al., "Potentialities of an electro-optic crystal fed by nuclear magnetic resonant coil for remote and low-invasive magnetic field characterization," IEEE Sensors Journal, vol. 13, no. 4, pp. 1274-1280, 2013.

[20] C. E. Hayes and L. Axel, "Noise performance of surface coils for magnetic resonance imaging at 1.5 T," Medical Physics, vol. 12, no. 5, pp. 604-607, 1985.

[21] E. Atalar, P. A. Bottomley, O. Ocali et al., "High resolution intravascular MRI and MRS by using a catheter receiver coil," Magnetic Resonance in Medicine, vol. 36, no. 4, pp. 596-605, 1996.

[22] D. Spence and M. Aimi, "Custom MEMS switch for MR surface coil decoupling," in Proceedings of the 23rd Annual Meeting of ISMRM, Toronto, Canada, April 2015.

[23] S. B. Bulumulla, K. J. Park, E. Fiveland, J. Iannotti, and F. Robb, "MEMS switch integrated radio frequency coils and arrays for magnetic resonance imaging," Review of Scientific Instruments, vol. 88, no. 2, Article ID 25003, 2017.

[24] F. Maggiorelli, E. B. Boskamp, G. Tiberi et al., "Double-tuned surface $1 \mathrm{H}-23 \mathrm{Na}$ radio frequency coils at $7 \mathrm{~T}$ : comparison of three decoupling methods," Applied Magnetic Resonance, vol. 50, no. 5, pp. 649-661, 2019.

[25] A. Maunder, M. Rao, F. Robb, and J. M. Wild, "Comparison of MEMS switches and PIN diodes for switched dual tuned RF coils," Magnetic Resonance in Medicine, vol. 80, no. 4, pp. 1746-1753, 2018.

[26] D. Darnell, Y. Ma, H. Wang, F. Robb, A. W. Song, and T.-K. Truong, "Adaptive integrated parallel reception, excitation, and shimming (iPRES-A) with microelectromechanical systems switches," Magnetic Resonance in Medicine, vol. 80, no. 1, pp. 371-379, 2018.

[27] K. Byron, S. A. Winkler, F. Robb, S. Vasanawala, J. Pauly, and G. Scott, "An MRI compatible RF MEMs controlled wireless power transfer system," IEEE Transactions on Microwave Theory and Techniques, vol. 67, no. 5, pp. 1717-1726, 2019.

[28] M. Fuentes, E. Weber, and S. Wilson, "Micro-electromechanical systems (MEMS) based RF-switches in MRI-a performance study," 2010.

[29] H. Raki, K. T. V. Koon, I. Saniour et al., "Serial and parallel active decoupling characterization using RF mems switches for receiver endoluminal coils at 1.5 T," IEEE Sensors Journal, vol. 20, no. 18, pp. 10511-10520, 2020.

[30] Feko Software, "Electromagnetic simulation software|Altair feko," 2020, https://altairhyperworks.com/product/FEKO. 
[31] B. K. Li, F. Liu, E. Weber, and S. Crozier, "Hybrid numerical techniques for the modelling of radiofrequency coils in MRI," NMR in Biomedicine, vol. 22, no. 9, pp. 937-951, 2009.

[32] R. Stara, N. Fontana, M. Alecci et al., "RF coil design for low and high field MRI: numerical methods and measurements," in 2011 IEEE Nuclear Science Symposium Conference Record, pp. 3465-3469, Valencia, Spain, October 2011.

[33] J.-J. Chung, R. C. Semelka, D. R. Martin, and H. B. Marcos, "Colon diseases: MR evaluation using combined T2-weighted single-shot echo train spin-echo and gadolinium-enhanced spoiled gradient-echo sequences," Journal of Magnetic Resonance Imaging, vol. 12, no. 2, pp. 297-305, 2000.

[34] D. I. Hoult and R. E. Richards, "The signal-to-noise ratio of the nuclear magnetic resonance experiment," Journal of Magnetic Resonance, vol. 24, no. 1, pp. 71-85, 1976.

[35] F. D. Doty, G. Entzminger, J. Kulkarni, K. Pamarthy, and J. P. Staab, "Radio frequency coil technology for small-animal MRI,” NMR in Biomedicine, vol. 20, no. 3, pp. 304-325, 2007.

[36] K. R. Minard and R. A. Wind, "Solenoidal microcoil design? part II: optimizing winding parameters for maximum signalto-noise performance," Concepts in Magnetic Resonance, vol. 13, no. 3, pp. 190-210, 2001.

[37] D. Kajfez, Q Factor Measurements, Analog and Digital, University of Missouri in Department of Electrical Engineering, Columbia, Missouri, 1999.

[38] D. Kajfez and E. J. Hwan, "Q-factor measurement with network analyzer," IEEE Transactions on Microwave Theory and Techniques, vol. 32, no. 7, pp. 666-670, 1984.

[39] G. Giovannetti, V. Hartwig, L. Landini, and M. F. Santarelli, "Low-field MR coils: comparison between strip and wire conductors," Applied Magnetic Resonance, vol. 39, no. 4, pp. 391-399, 2010.

[40] G. Giovannetti, V. Hartwig, L. Landini, and M. F. Santarelli, "Sample-induced resistance estimation in magnetic resonance experiments: simulation and comparison of two methods," Applied Magnetic Resonance, vol. 40, no. 3, pp. 351-361, 2011.

[41] G. Giovannetti, "Comparison between circular and square loops for low-frequency magnetic resonance applications: theoretical performance estimation," Concepts in Magnetic Resonance Part B: Magnetic Resonance Engineering, vol. 46B, no. 3, pp. 146-155, 2016.

[42] G. Giovannetti, V. Viti, and Y. Liu, "An accurate simulator for magnetic resonance coil sensitivity estimation," Concepts in Magnetic Resonance Part B: Magnetic Resonance Engineering, vol. 33B, pp. 209-215, 2008.

[43] M. Armenean, O. Beuf, and F. Pilleul, "Optimization of endoluminal loop radiofrequency coils for gastrointestinal wall MR imaging," IEEE Sensors Journal, vol. 4, no. 1, pp. 57-64, 2004. 